\title{
Éditorial
}

\section{Contrôle judiciaire dans l'usine à lois européenne}

L'Union européenne est en passe d'adhérer à la convention européenne des droits de l'homme, ce qui pourrait profiter à la protection des droits fondamentaux en Europe. Cela fait bien longtemps que, dans une zone particulièrement sensible à la thématique des droits fondamentaux - l'« espace de la liberté, de la sécurité et de la justice »-, divers signaux indiquent clairement qu'une focalisation exclusive sur les intérêts exécutifs de la sécurité intérieure est pour le moins ternie : les droits fondamentaux dans les procédures pénales, mais aussi la protection des données doivent être renforcés au niveau européen. L'usine à lois européenne produit : plus de lois européennes axées sur la protection des droits fondamentaux, plus de recommandations politiques visant à développer la protection juridique institutionnelle. Plans de sauvetage mis à part, l'Union européenne semble vouloir célébrer en grande pompe la « joie de la belle étincelle divine » de la liberté et de la protection juridique. Mais l'étincelle en question ne dégagerait-elle qu'une belle lumière? Les expériences vécues avec les pouvoirs politiques et administratifs en Europe prouvent encore le contraire. Les articles de ce numéro, principalement consacré au contrôle judiciaire dans l'usine à lois européenne, émettent quelques doutes quant à l'aboutissement de l'institutionnalisation et de la mise en œuvre des mécanismes judiciaires de protection et quant à savoir si elles peuvent et doivent aboutir.

Ainsi, Henri Labayle fait état d'un processus de judiciarisation qui entend certes d'une part établir formellement les droits fondamentaux dans l'espace pénal européen, mais qui ne dispose d'autre part d'aucune protection suffisante dans le système juridique européen. L'usine à lois produit toujours plus de réglementations, mais abandonne celles-ci à une mise en œuvre artisanale et diversifiée par chacun des États membres. Ce genre d'usine à lois perd essentiellement - et encore - de vue la protection de l'individu. L'article d'Erhard Denninger thématise les manquements de la protection juridique européenne en elle-même : l'interprétation des normes européennes n'est pas toujours motivée par le meilleur épanouissement possible des droits individuels, mais imprégnée par les " conceptions prédéterminées de politique juridique communautaire ». L'intégration systémique passe ainsi avant la liberté individuelle. Gavin Robinson énonce clairement que la forme hiérarchique classique du contrôle judiciaire doit avorter, compte tenu de la mise en réseau des données, de leur sauvegarde et de leur traitement par des tiers : le contrôle social, qui se dissocie des États, mais également d'une association d'États, requiert un renforcement de la protection juridique commune à l'Union européenne. L'urgence de ce processus est mise en exergue par l'article de Sandro Dicker : la décision d'instruction européenne entend dépasser les systèmes nationaux d'interdiction de preuves, sans garantir toutefois que le niveau de protection des droits fondamentaux dans les procédures pénales soit identique dans l'ensemble de l'Union, et ce dans le détail. 
Au-delà du sujet principal de ce numéro, il est également question de domaines extrêmement sensibles à la thématique des droits fondamentaux, qui ont pour objet la protection des individus contre le pouvoir administratif ou même religieux. Dirk Wüstenberg demande que, dans le cadre de la modification de l'article 226 du Code pénal allemand (StGB), l'état de fait des « mutilations génitales » soit remplacé par l'état de fait d" " organe », afin de satisfaire aux directives juridiques des Nations unies et de l'Organisation mondiale de la santé. Pour terminer, Astrid Wallrabenstein requiert une refonte conforme à la Constitution de la loi sur les prestations sociales des demandeurs d'asile qui ne peut se contenter d'un statu quo.

Nous espérons que ce numéro pourra apporter une contribution critique constructive à la production de lois dans l'usine à lois européenne.

Luxembourg, décembre 2012

Stefan Braum 